\title{
Chiral unitary model for the kaonic atom
}

\author{
S. Hirenzaki, ${ }^{1}$ Y. Okumura, ${ }^{1}$ H. Toki, ${ }^{2}$ E. Oset, ${ }^{2,3}$ and A. Ramos ${ }^{4}$ \\ ${ }^{1}$ Department of Physics, Nara Women's University, Nara 630-8506, Japan \\ ${ }^{2}$ Research Center for Nuclear Physics (RCNP), Osaka University, Ibaraki, Osaka 567-0047, Japan \\ ${ }^{3}$ Departamento de Física Teórica, Universidad de Valencia, 46100 Burjassot (Valencia), Spain \\ ${ }^{4}$ Departament d'Estructura i Constituents de la Materia, Universidad de Barcelona, 08028 Barcelona, Spain
}

(Received 8 November 1999; published 11 April 2000)

\begin{abstract}
We study kaonic atoms over the periodic table using a kaon self-energy in the nuclear medium derived from the SU(3) chiral unitary model. This model is quite successful in reproducing the scattering amplitude of meson meson and the strangeness $S=-1$ meson baryon reactions. In particular the properties of the $\Lambda$ (1405) resonance are well reproduced. In the nuclear medium the properties of this resonance are appreciably modified, and consequently the kaon nucleon scattering amplitudes, leading to an attractive kaon nucleus selfenergy for densities higher than $\rho_{0} / 25$. With this interaction we are able to reproduce shifts and widths of kaonic atoms over the periodic table. We also investigate the region of deeply bound kaonic nuclear states which appear with very large widths in medium and heavy nuclei. Some of the deep atomic states, still unobserved, appear with narrower widths than the separation between levels, which makes them eligible for experimental observation. To this end, we make some estimates of the rates of formation in the $\left(K^{-}, \gamma\right)$ reaction.
\end{abstract}

PACS number(s): 13.75.Jz, 36.10.-k, 25.80.Nv

\section{INTRODUCTION}

Kaonic atoms carry important information of the $K^{-}$-nucleon interaction in a nuclear medium. This information is very important for the constraints of kaon condensation in high density matter. The properties of kaons in nuclei are largely influenced by the change of the $\Lambda(1405)$ in the nuclear medium, since $\Lambda(1405)$ is a resonance state just below the kaon nucleon threshold.

In fact, there were studies of kaonic atoms by modifying the properties of $\Lambda(1405)$ in the nuclear medium [1-3]. These works demonstrated the ability of reproducing the kaonic atom properties, which come out to be as good as the phenomenological study of Batty [4].

Recently, there have been very important developments in the description of hadron properties in terms of the SU(3) chiral Lagrangian. The unitarization of the chiral Lagrangian provides the $\Lambda(1405)$ resonance state as a baryon-meson coupled system $[5,6]$. It is particularly interesting to mention that the use of the complete set of meson-baryon states that can be built from the states of the SU(3) flavor octets of baryons and mesons, together with a suitable cutoff in the loops, is able to provide the $\Lambda(1405)$ resonance with only the lowest order chiral coupling terms [6].

It is then very natural to study the properties of $\Lambda(1405)$ in the nuclear medium using the SU(3) chiral unitary model. This work has been performed by Waas et al. [7], Lutz [8], and Ramos and Oset [9]. All of them have considered the Pauli effect on the intermediate nucleons. In addition in [8] the self-energy of the kaon in the intermediate states is considered and in [9] the self-energy of the pions and baryons is also taken into account. These approaches lead to a kaon self-energy in the nuclear medium which can be tested with $K^{-}$atoms.

In this work, we take the scattering amplitude in the nuclear medium calculated by Ramos and Oset [9] for the case of kaonic atoms. First of all, we demonstrate the ability of reproducing the existing kaonic atom data as the optical potential of Batty [4]. We then calculate the deeply bound kaonic atoms for ${ }^{16} \mathrm{O}$ and ${ }^{40} \mathrm{Ca}$, which are found to have narrow widths and are expected to be observed by proper methods. We also get very deep kaonic nuclear states, which have large decay widths of the order of several tens of $\mathrm{MeV}$.

Since the standard method of measuring the kaonic atoms is limited to those atomic states with small widths, less than $\Gamma \leqslant 1 \mathrm{keV}$, other methods would have to be explored. Recently, we have witnessed the usefulness of the direct reactions for the formation of the deeply bound pionic atoms by using the $\left(d,{ }^{3} \mathrm{He}\right)$ reactions [10-12]. However, in the present case one would have to produce a $K^{+}$in addition and there would be a large momentum mismatch. For this reason the $\left(K^{-}, \gamma\right)$ reaction would be more suitable and we have calculated the rates for the formation of kaonic atoms and deeply bound kaonic nuclear states on ${ }^{40} \mathrm{Ca}$ using the formulation developed for the formation of deeply bound pionic atoms with the $\left(\pi^{-}, \gamma\right)$ reaction in [13].

This paper is arranged as follows. In Sec. II we sketch the chiral unitary model for the calculation of the kaon-nucleon scattering amplitude in the medium. This amplitude provides the kaon-nucleus optical potential for kaonic atoms, which is discussed in Sec. III. In Sec. IV we present the numerical results for kaonic atoms. The results are compared with those with phenomenological potentials and with experimental data. We study also the deeply bound kaonic nuclear states for ${ }^{40} \mathrm{Ca}$. We calculate the $\left(K^{-}, \gamma\right)$ cross sections for the formation of kaonic atoms and deeply bound kaonic nuclear states in Sec. V. Finally, Sec. VI is devoted to a summary of this paper.

\section{CHIRAL UNITARY MODEL FOR THE KAONIC ATOM POTENTIAL}

We start with the construction of the kaon-nucleon scattering amplitude in the nuclear medium. The microscopic 


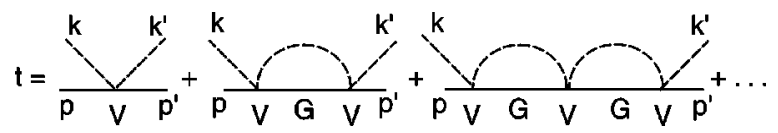

FIG. 1. Diagrammatic representation of the Bethe-Salpeter equation in $\bar{K} N$ scattering.

evaluation of the kaonic atom optical potential is based on the chiral unitary model of Ref. [6]. This work follows closely the steps of Ref. [5], where the usefulness of combining unitarity with the chiral Lagrangian was made manifest. With respect to Ref. [5], the work of [6] introduces the complete set of states of the pseudoscalar octet of mesons and the octet of stable baryons, while only the channels open at low energies were included in [5]. The inclusion of all the channels allows one to obtain good solutions in [6] by means of only the lowest order chiral Lagrangian and a suitable cut off to regularize the loop integrals, while in Ref. [5] the effect of the unopen channels was replaced by the inclusion of higher order terms in the chiral Lagrangian.

The $K^{-} N t$ matrix is obtained in [6] through the iteration of the lowest order Lagrangian in a coupled channel BetheSalpeter equation

$$
t_{i j}=V_{i j}+V_{i k} G_{k} t_{k j}
$$

where $V$ and $t$ appear to be factorized with their on-shell values in the $V G t$ combination, and $G$ is the loop integral of meson and baryon propagators. Diagrammatically the series implicit in Eq. (1) is shown in Fig. 1.

The $K^{-}$self-energy is evaluated in detail in Ref. [9] for nuclear matter by means of the integral

$$
\Pi_{K}(k, \rho)=2 \sum_{N} \int \frac{d^{3} p}{(2 \pi)^{3}} n(\vec{p}, \rho) t_{K^{-}{ }_{N}}^{(m)}(k, \vec{p}, \rho),
$$

where $N$ stands for protons or neutrons and $t^{(m)}$ is the $K^{-} N$ scattering matrix in the nuclear medium with density $\rho$. In Eq. (2), $n(\vec{p}, \rho)$ denotes the occupation probability of momentum states in the Fermi sea in the nuclear medium at finite density $\rho$. Only the $s$-wave amplitude is considered, which is the one of relevance for $K^{-}$atoms.

The $t^{(m)}$ matrix is evaluated with the same equation (1) but modifications are done in the meson and baryon propagators of the loops to incorporate the medium effects. The states allowed in the loops are $\bar{K} N, \pi \Sigma, \pi \Lambda, \eta \Sigma, \eta \Lambda$, and $K \Xi$.

The modifications are the following.

(1) Pauli blocking in the nucleon propagators. This effect was proved to be very important in $[7,14]$. Since now one needs to place the intermediate nucleon states on top of a Fermi sea, this costs more energy and the net effect is a shift to higher energies of both the real and imaginary parts of the $\Lambda$ (1405) dominated $K^{-} p$ amplitude. The shift of the real part automatically produces an attractive $K^{-}$self-energy already at very small densities.

(2) However, if the $K^{-}$self-energy acquires a negative value, then it costs less energy to produce the $\Lambda$ (1405) resonance and this produces a shift of the $K^{-} p$ amplitude to-

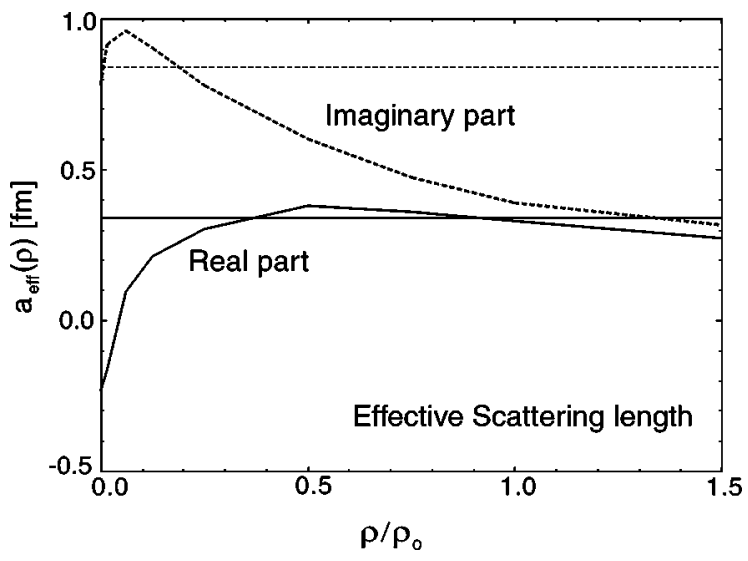

FIG. 2. The effective scattering length $a_{e f f}$, defined by Eq. (3) in the text, is shown as a function of the nuclear density divided by the normal density. The thick solid line (real part) and the thick dashed line (imaginary part) are the results of the chiral unitary model. The thin lines are those of the phenomenological fit to kaonic atom data [4].

wards lower energies. This means that a self-consistent evaluation becomes necessary here and this was first proved in [8], where it was found that the consideration of the $K^{-}$ self-energy together with Pauli blocking on the nucleons led to modifications of the $\Lambda(1405)$, mostly on the imaginary part (the resonance becomes wider), while the position was barely changed. In Ref. [9] the $K^{-}$self-energy is also considered and a self-consistent evaluation is also done.

(3) In addition to the former ingredients new effects are considered in [9]; i.e., the pion self-energy in the $\pi \Sigma, \pi \Lambda$ channels is also taken into account, allowing the pions to excite $p h, \Delta h$, and $2 p 2 h$ components. The difference of binding between the nucleons and $\Sigma$ or $\Lambda$ is also incorporated.

The results obtained are qualitatively similar to those found in [8] except that the imaginary part of the $K^{-} p$ amplitude becomes even wider and essentially flattens at full nucleon density $\rho \sim \rho_{0}$.

The $K^{-}$self-energy obtained in [9] from the interaction of the $K^{-}$with protons and neutrons in symmetric nuclear matter at $K^{-}$threshold $\left(k^{0}=m_{K}, \vec{k}=0\right)$ is parametrized here in terms of a density dependent complex effective $K^{-}$scattering length, such that

$$
\Pi_{K}(k, \rho)=-4 \pi \eta a_{e f f}(\rho) \rho,
$$

with $\eta=1+m_{K} / M_{N}$ and $\rho\left(\vec{p}_{F}\right)=\left(2 / 3 \pi^{2}\right)\left|\vec{p}_{F}\right|^{3}$, in order to facilitate a comparison with the effective scattering length derived from fits to the $K^{-}$atom data. The results are shown in Fig. 2.

One can see in Fig. 2 that Re $a_{\text {eff }}$ passes from negative to positive (repulsive to attractive) from $\rho=0$ to $\rho \geqslant \rho_{0} / 25$. Thus, the chiral unitary model provides the attractive kaonnucleus optical potential naturally while keeping the repulsive sign for the kaon-nucleon scattering length in the free space. In Fig. 2, we also show the phenomenological scattering length obtained by Batty [4] from fits to the kaonic atom data. We find that both results are similar at $\rho \sim \rho_{0} / 4$. Since 
the kaonic atom data are also reproduced well by the chiral unitary model, as we will see later on, it seems that the strong shifts and widths of the kaonic atoms are mainly determined by the optical potential strength at a certain nuclear density, $\rho \sim \rho_{0} / 4$. This was the case of pionic atoms [15] although the effective densities felt by the pions were different.

We would like to mention other density dependent scattering lengths obtained by the phenomenological fit of [16]. One of their results can be written as

$$
a_{\text {eff }}(\rho)=(-0.15+0.62 i)+(1.66-0.04 i)\left[\rho / \rho_{0}\right]^{0.24}[\mathrm{fm}] .
$$

The real part of this effective scattering length also changes its sign and provides an attractive interaction in the nuclear medium. However, the real part depends on the density much more strongly than our results and gives $\operatorname{Re} a_{\text {eff }}\left(\rho_{0}\right)$ $=1.51[\mathrm{fm}]$. On the other hand, the density dependence of the imaginary part is rather flat and the strength is similar to our results.

\section{KAONIC ATOM STRUCTURE}

We study the properties of kaonic bound states by solving the Klein-Gordon equation

$$
\left[-\vec{\nabla}^{2}+\mu^{2}+2 \mu V_{\text {opt }}(r)\right] \phi(\vec{r})=\left[E-V_{\text {Coul }}(r)\right]^{2} \phi(\vec{r}) .
$$

Here, $\mu$ is the kaon-nucleus reduced mass and $V_{C o u l}(r)$ is the Coulomb potential with a finite nuclear size:

$$
V_{\text {Coul }}(r)=-e^{2} \int \frac{\rho_{p}\left(r^{\prime}\right)}{\left|\vec{r}-\vec{r}^{\prime}\right|} d^{3} r^{\prime},
$$

where $\rho_{p}(r)$ is the proton density distribution. We take the Woods-Saxon form for the density and keep the shapes of neutron and proton density distributions the same:

$$
\rho(r)=\rho_{n}(r)+\rho_{p}(r)=\frac{\rho_{0}}{1+\exp [(r-R) / a]},
$$

where we use $R=1.18 A^{1 / 3}-0.48[\mathrm{fm}]$ and $a=0.5[\mathrm{fm}]$ with $A$ the nuclear mass number.

The kaon-nucleus optical potential is related to the kaon self-energy in nuclear matter as $\Pi_{K}=2 \mu V_{o p t}$. We use the optical potential in a finite nucleus. In coordinate space this is accomplished by means of the local density approximation (LDA), where $\rho$ of nuclear matter is substituted by $\rho(r)$ of the nucleus. This procedure is exact for the lowest order term in the density of the $S$-wave self-energy and arguments were given in [17] for the accuracy of the LDA in higher orders. At the same time the translation code from nuclear matter to finite nuclei for $P$ waves was also given. Thus, we have

$$
2 \mu V_{o p t}(r)=-4 \pi \eta a_{e f f}(\rho) \rho(r),
$$

with the effective scattering length $a_{\text {eff }}$ and $\eta$ defined in Sec. II.

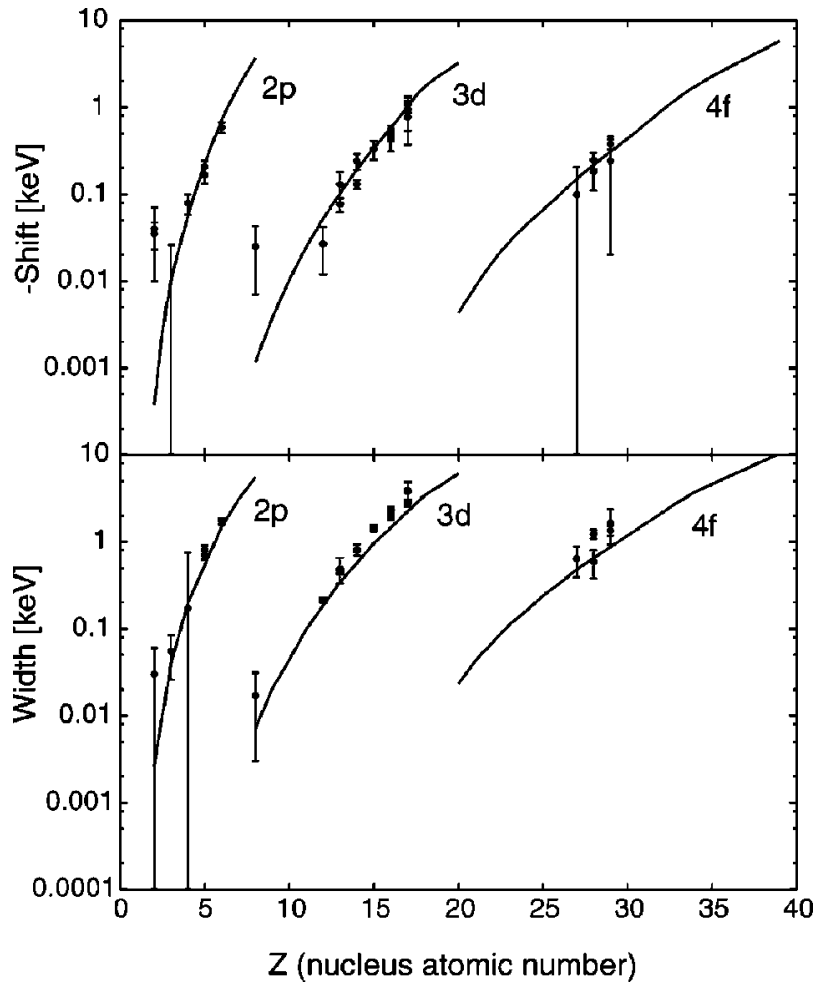

FIG. 3. The calculated energy shifts and widths are shown as functions of the nucleus atomic number for $2 p, 3 d$, and $4 f$ kaonic atom states. Experimental data are also shown [20].

We solve the Klein-Gordon equation numerically following the method of Oset and Salcedo [18]. The applications of the method to the pionic atom studies were reported in detail in Ref. [19].

\section{NUMERICAL RESULTS}

We show here the numerical results on kaonic atoms with the optical potential obtained from the local density approximation using the chiral unitary selfenergy at various nuclear matter densities. We show in Fig. 3 the energy shifts and widths for several kaonic atoms in comparison with data. The calculated results agree with the experimental data well. The quality of the agreement is as good as the phenomenological potentials. We note, here, that the theoretical model does not contain any free parameter to reproduce the data.

The energy levels for atomic kaonic states in $\mathrm{O}$ and $\mathrm{Ca}$ are shown in Fig. 4, where the results of the chiral model and those of the phenomenological model [Eq. (4)] are compared. We can see that the results obtained with both potentials are very similar. We find that the deep atomic states such as $1 s$ in ${ }^{40} \mathrm{Ca}$, still unobserved, appear with narrower widths than the separation between levels and are predicted to be quasistable states. Similar results to those of the phenomenological potential shown in Fig. 4 were reported by Friedman and Gal [21].

In Fig. 5, we show the energy levels including the shallow atomic and the deep nuclear kaonic states of $\mathrm{Ca}$ using the chiral unitary model potential. The shallow atomic states are shown by dashed bars. The deep nuclear ones are shown by 


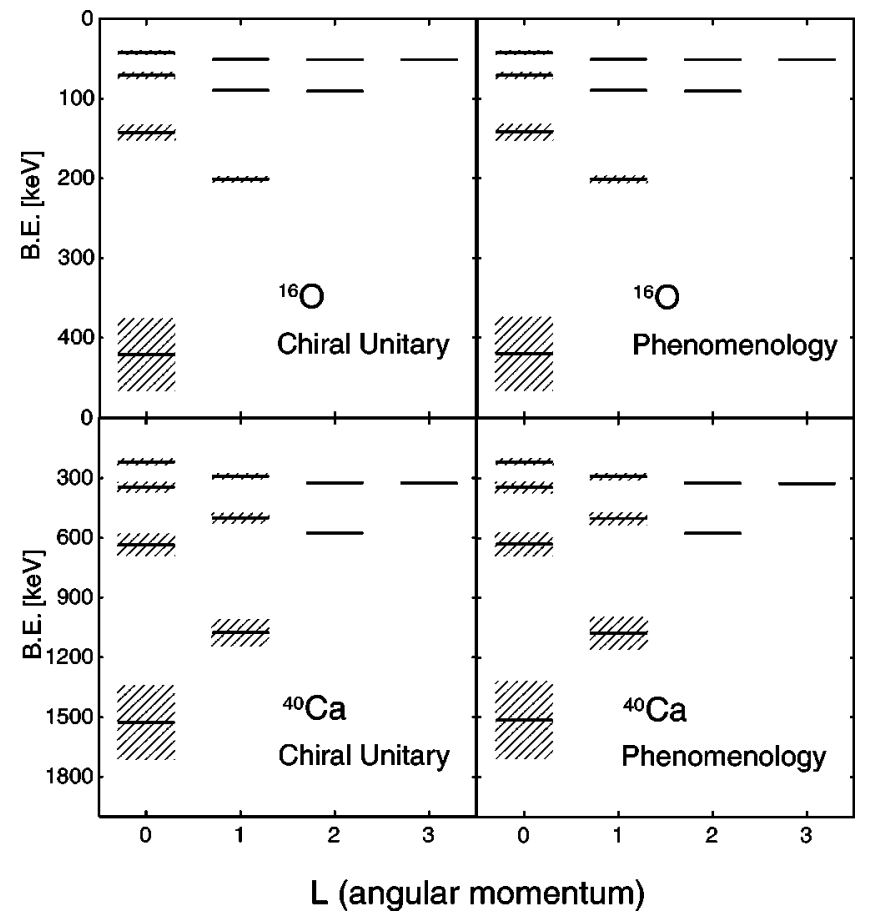

FIG. 4. Calculated energy levels for kaonic atoms in ${ }^{16} \mathrm{O}$ and ${ }^{40} \mathrm{Ca}$. The hatched area indicates the widths of the states. Results with the chiral unitary model (left) and those with the phenomenological potential, defined in Eq. (4) (right), are compared.

solid bars with the numbers, which indicate the widths in units of $\mathrm{MeV}$. These nuclear states have extremely large widths and will not be observed as peak structures in experiments.

Wave functions of $1 s, 2 p$, and $2 s$ kaonic atom states in $\mathrm{Ca}$ are shown in Fig. 6 for the cases with and without the strong interaction of the chiral unitary model. The finite-size

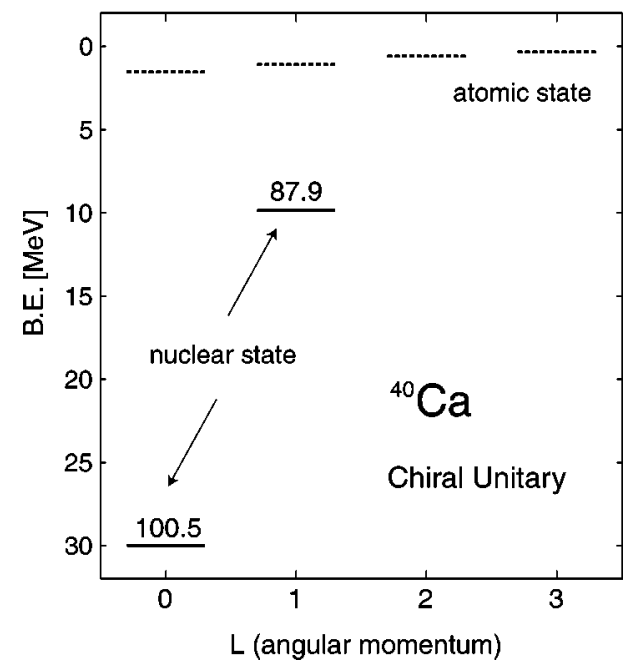

FIG. 5. The energy levels of the nuclear kaonic states of ${ }^{40} \mathrm{Ca}$ are shown for the $L=0(s)$ and $L=1(p)$ states. As a reference, the yrast levels of the atomic kaonic states are shown by dashed lines up to $L=3$. The atomic levels above the yrast levels are shown in Fig. 4 . The numbers above the solid lines indicate the full widths in $\mathrm{MeV}$.

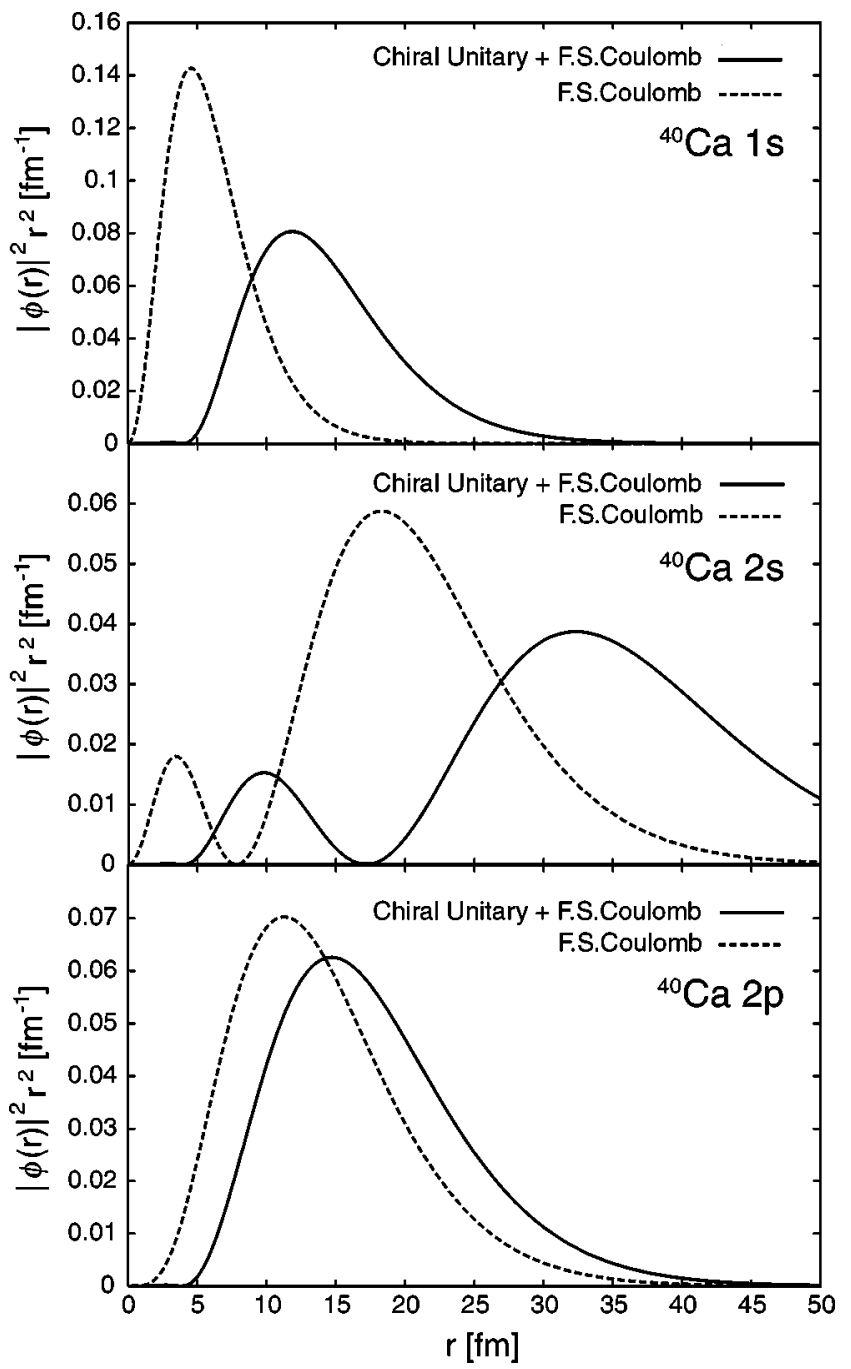

FIG. 6. Wave functions of $1 s, 2 p$, and $2 s$ kaonic atom states in ${ }^{40} \mathrm{Ca}$ are shown. The dashed curves are the wave functions calculated with the finite-size Coulomb potential. The solid lines are those with the strong interaction of the chiral unitary model and the finite-size Coulomb potential.

Coulomb potential provides the wave functions depicted by dashed curves, which are pushed outwards by the strong interaction.

The wave functions of the deep nuclear kaonic states are depicted in Fig. 7 for the $s$ and $p$ states in $\mathrm{Ca}$. The wave functions stay almost inside of the nuclear radius, which is about $3.5 \mathrm{fm}$ for $\mathrm{Ca}$. Hence, the widths become extremely large, of the order of $100 \mathrm{MeV}$.

\section{KAONIC ATOM FORMATION}

Usually the information on the kaonic atoms are obtained by the kaonic $\mathrm{x}$ rays after trapping slow negative kaons. In this case, deeper kaonic states below certain levels are not populated due to the kaonic absorption by the nucleus. Hence, we have to find another method to excite the kaonic states. The natural reaction to start with is the radiative kaonic capture reaction $\left(K^{-}, \gamma\right)$. This type of reaction was studied in the context of the deeply bound pionic atom for- 


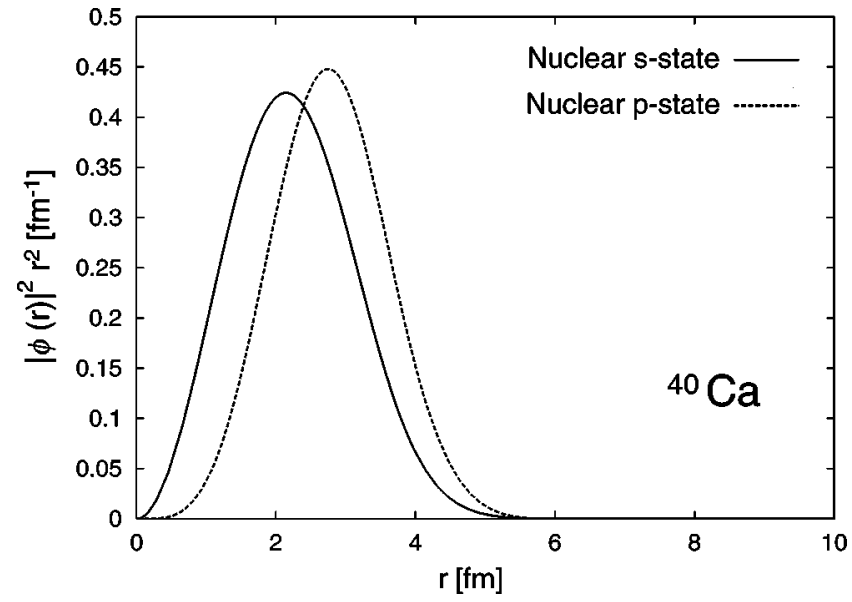

FIG. 7. The wave functions of the interior kaonic nuclear states are shown for the $s$ and $p$ states in ${ }^{40} \mathrm{Ca}$.

mation in Ref. [13]. We use the same theoretical model to calculate the formation cross section, which is expressed as

$$
\begin{aligned}
\frac{d^{2} \sigma}{d \Omega d k}= & \frac{1}{(2 \pi)^{3}} \frac{k}{q} e^{2}|\vec{q}|^{2} \sin ^{2} \Theta \\
& \times \frac{\Gamma_{n l}}{\left[\omega(q)+M_{A}-k-E\left(A K^{-}\right)\right]^{2}+\left(\Gamma_{n l} / 2\right)^{2}} \\
& \times \frac{1}{2 \omega_{K}} \sum_{m}|T|^{2}, \\
T & =\int d^{3} x \exp [i(\vec{q}-\vec{k}) \cdot \vec{x}] \Phi_{n l m}^{*}(\vec{x}),
\end{aligned}
$$

TABLE I. Formation cross sections of kaonic atoms on ${ }^{16} \mathrm{O}$ and ${ }^{40} \mathrm{Ca}$ in $\left(K^{-}, \gamma\right)$ reactions at $T_{K}=20 \mathrm{MeV}$. Each cross section has a peak at the emitted photon energy $E_{\gamma}=T_{K}+B$, where $B$ is the binding energy of each state shown in the table in parentheses in units of $\mathrm{MeV}$.

\begin{tabular}{cccc}
\hline \hline cuc & $\begin{array}{c}{\left[K^{-}{ }^{16} \mathrm{O}\right]} \\
{[\mu \mathrm{b} /(\mathrm{sr} \mathrm{MeV})]}\end{array}$ \\
{$[\mathrm{deg}]$} & $1 s(0.41)$ & $2 p(0.20)$ & $2 s(0.14)$ \\
\hline 20 & 0.113 & 0.216 & 0.113 \\
40 & 0.323 & 0.674 & 0.326 \\
60 & 0.425 & 1.026 & 0.429 \\
80 & 0.383 & 1.078 & 0.388 \\
& \multicolumn{3}{c}{} \\
& $d^{2} \sigma / d k d \Omega$ & {$[\mu \mathrm{b} /(\mathrm{sr} \mathrm{MeV})]$} & \\
{$[\mathrm{deg}]$} & $1 s(1.53)$ & $2 p(1.08)$ & $2 s(0.63)$ \\
\hline 20 & 0.112 & 0.471 & 0.115 \\
40 & 0.294 & 1.431 & 0.307 \\
60 & 0.352 & 2.061 & 0.371 \\
80 & 0.292 & 2.019 & 0.309 \\
\hline \hline
\end{tabular}

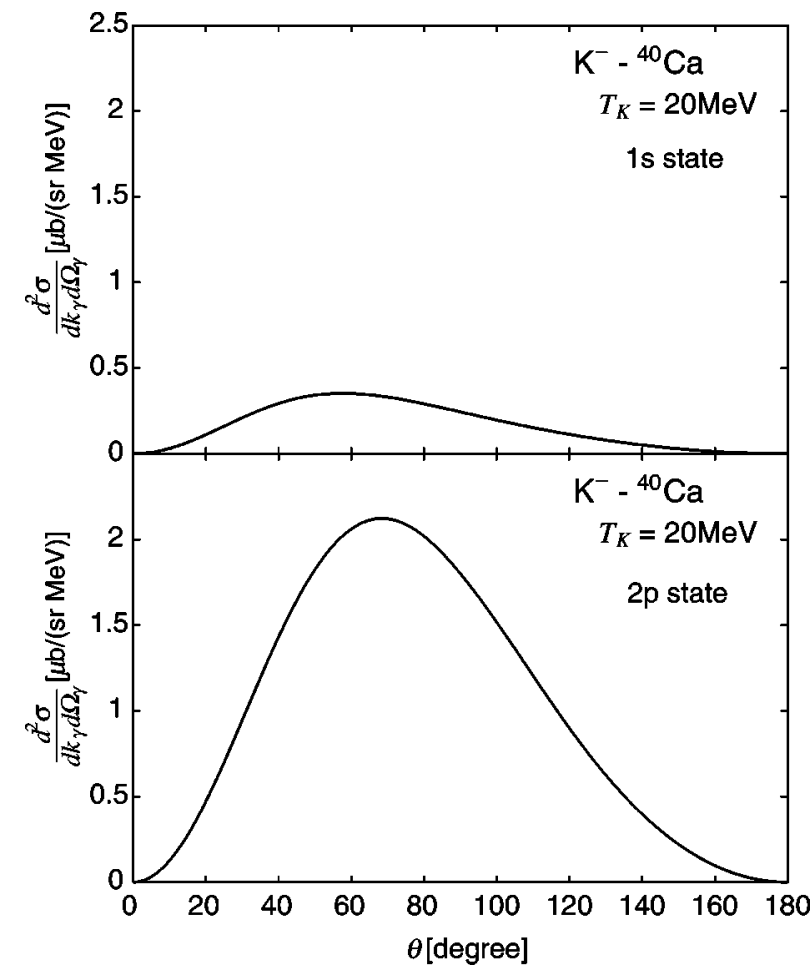

FIG. 8. The double differential cross section at the peak of the photon energy distribution is plotted as a function of the emitted photon angle in the laboratory frame for the ${ }^{40} \mathrm{Ca}\left(K^{-}, \gamma\right)$ reaction leading to kaonic atoms at $T_{K}=20 \mathrm{MeV}$.

where $q$ and $k$ are the incoming kaon and the emitted photon momenta, $e^{2} / 4 \pi=1 / 137, \Theta$ the angle between $\vec{q}$ and $\vec{k}$, $\omega(q)$ the incoming kaon energy, $\omega_{K}$ the bound kaon energy, $\Gamma_{n l}$ the width of the kaonic state, and $\Phi_{n l m}$ its wave function. $M_{A}$ is the mass of the initial nucleus and $E\left(A K^{-}\right)$the energy of final nucleus with the bound kaon. We neglect the recoil energy of the nucleus and approximate the incident kaon

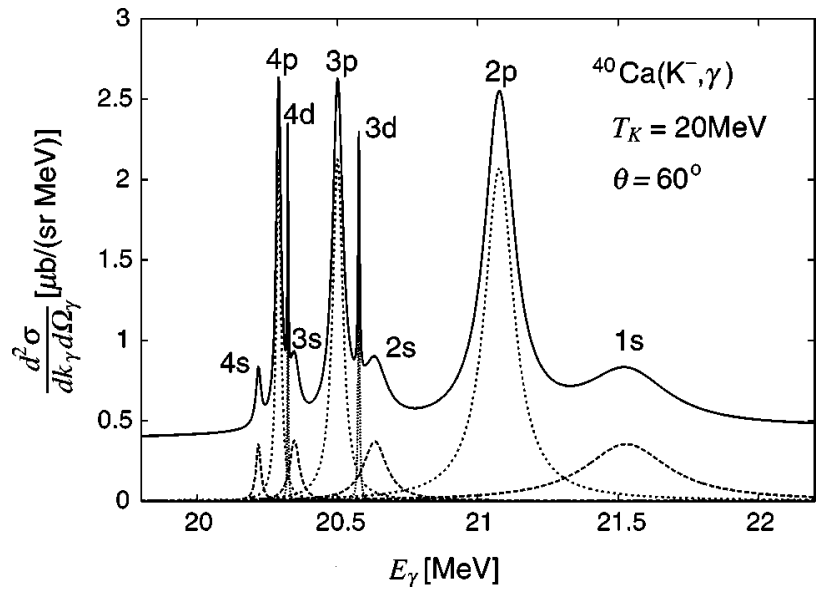

FIG. 9. The double differential cross sections leading to kaonic atoms for ${ }^{40} \mathrm{Ca}$ are plotted as function of the energy of the emitted photon at $T_{K}=20 \mathrm{MeV}$ and $\theta_{l a b}=60^{\circ}$. Each contribution of atomic state is shown by a dotted or dashed line. The contributions from nuclear kaonic states, which are almost constant in this energy range, are also included in the sum of all contributions (solid line). 


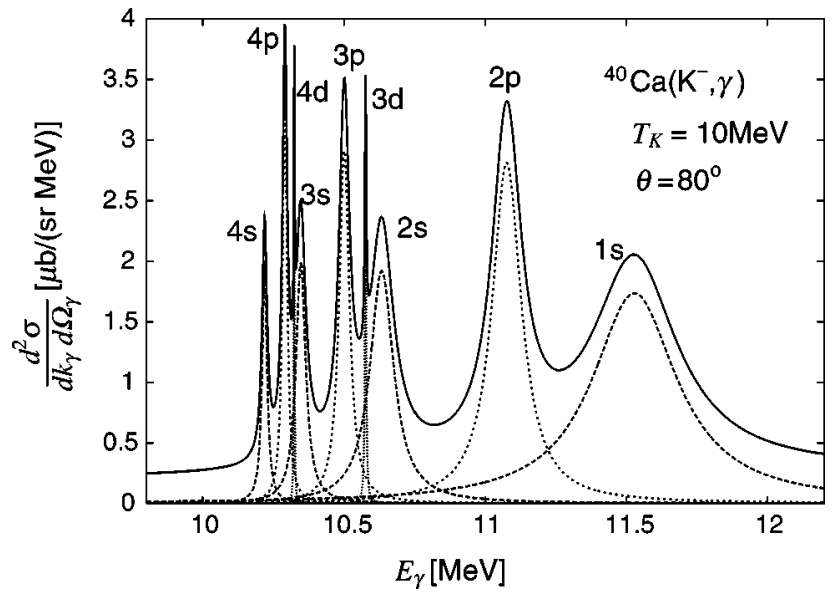

FIG. 10. Same as Fig. 9 except for $T_{K}=10 \mathrm{MeV}$ and $\theta_{\text {lab }}$ $=80^{\circ}$.

wave by a plane wave. This latter approximation is supposed to overstimate the rate of formation but the order of magnitude obtained should still be fine.

We show the calculated angular distributions of $\left(K^{-}, \gamma\right)$ reaction leading to kaonic atoms for $T_{K}=20 \mathrm{MeV}$ and for $E_{\gamma}=T_{K}+B$ with $B$ the binding energy of the atoms in Table I and Fig. 8. The peaks appear around $60^{\circ}$ for both the $1 s$ and $2 p$ excitation cross sections.

The double differential cross sections leading to kaonic atoms at $T_{K}=20 \mathrm{MeV}$ for $\mathrm{Ca}$ are shown in Fig. 9 for $\theta$ $=60^{\circ}$. In this case all the $p$ states are excited very clearly and could be observed in experiments provided enough resolution can be reached for the photon detectors. The solid curve shows the sum of all contributions including the contributions from the formation of the deep kaonic nuclear states. The result at $T_{K}=10 \mathrm{MeV}$ is shown in Fig. 10. By lowering the incident kaon energy, we can reduce the momentum transfer and observe the $1 s$ state more clearly. We should mention also that the background coming from the nuclear kaonic states is reduced.

\section{CONCLUSION}

We have studied the kaonic atoms using the optical potential obtained by the chiral unitary model. The chiral unitary model is able to provide the $\Lambda(1405)$ resonance state in free space as the coupled channel state of flavor octet baryon and meson states. The chiral unitary model has been used then to calculate the $K^{-} p$ scattering amplitudes as a function of density in the nuclear medium by taking into account the medium effects on the baryons and the mesons. Hence, the scattering amplitudes thus obtained do not contain any free parameter. We have constructed the kaon-nucleus optical potential from the $\mathrm{K}^{-}$self-energy in nuclear matter by using the local density approximation.

We have calculated first the shallow kaonic atom states in a wide mass range and compared with the experimental data and also with the calculated results of Batty and co-workers $[4,20]$. The agreement is very satisfactory.

We have then calculated all the kaonic states including the deeply bound kaonic nuclear states for $\mathrm{Ca}$. We see that these deeply bound kaonic nuclear states have very large widths.

We have made some estimates for the formation cross sections of kaonic atoms with the $\left(K^{-}, \gamma\right)$ reaction. We hope this reaction can be used for the study of kaonic atoms and deeply bound kaonic nuclear states.

\section{ACKNOWLEDGMENTS}

E.O. is thankful for the support of Monbusho, which enabled him to stay at RCNP in Osaka University, where this work was performed. S.H. and Y.O. acknowledge A. Gal and E. Friedman for comments and suggestions on numerical calculation for kaonic bound states. S.H. and Y.O. also thank J. Nieves for kindly providing us with his pionic atom code. We would like to acknowledge support from the EU, TMR Network Eurodaphne, Contract No. ERBFMRX-CT98-0169.
[1] M. Alberg, E. M. Henley, and L. Wilets, Ann. Phys. (N.Y.) 96, 43 (1976).

[2] R. Brockmann, W. Weise, and L. Tauscher, Nucl. Phys. A308, 365 (1978)

[3] M. Mizoguchi, S. Hirenzaki, and H. Toki, Nucl. Phys. A567, 893 (1994).

[4] C. J. Batty, Nucl. Phys. A372, 418 (1981).

[5] N. Kaiser, P. B. Siegel, and W. Weise, Nucl. Phys. A594, 325 (1995).

[6] E. Oset and A. Ramos, Nucl. Phys. A635, 99 (1998).

[7] T. Waas, N. Kaiser, and W. Weise, Phys. Lett. B 365, 12 (1996); 379, 34 (1996); T. Waas and W. Weise, Nucl. Phys. A625, 287 (1997).

[8] M. Lutz, Phys. Lett. B 426, 12 (1998).

[9] A. Ramos and E. Oset, Nucl. Phys. A (to be published).

[10] H. Toki and T. Yamazaki, Phys. Lett. B 213, 129 (1988); H. Toki, S. Hirenzaki, R. S. Hayano, and T. Yamazaki, Nucl. Phys. A501, 653 (1989).
[11] S. Hirenzaki, H. Toki, and T. Yamazaki, Phys. Rev. C 44, 2472 (1991); H. Toki, S. Hirenzaki, and T. Yamazaki, Nucl. Phys. A530, 679 (1991).

[12] T. Yamazaki et al., Z. Phys. A 355, 219 (1996); Phys. Lett. B 418, 246 (1998).

[13] J. Nieves and E. Oset, Phys. Lett. B 282, 24 (1992).

[14] V. Koch, Phys. Lett. B 337, 7 (1994).

[15] R. Seki and K. Masutani, Phys. Rev. C 27, 2799 (1983).

[16] C. J. Batty, E. Friedman, and A. Gal, Phys. Rep. 287, 385 (1997).

[17] J. Nieves and E. Oset, Nucl. Phys. A554, 509 (1993).

[18] E. Oset and L. L. Salcedo, J. Comput. Phys. 57, 361 (1985).

[19] J. Nieves, E. Oset, and C. Garcia-Recio, Nucl. Phys. A554, 509 (1993).

[20] E. Friedman, A. Gal, and C. J. Batty, Nucl. Phys. A579, 518 (1994).

[21] E. Friedman and A. Gal, Phys. Lett. B 459, 43 (1999). 